\title{
Does a Spin-Orbit Coupling Between the Sun and the Jovian Planets Govern the Solar Cycle?
}

\author{
I. R. G. Wilson ${ }^{\mathrm{A}, \mathrm{C}}$, B. D. Carter $^{\mathrm{B}}$, and I. A. Waite $^{\mathrm{B}}$ \\ ${ }^{\text {A }}$ Education Queensland, Toowoomba, QLD 4350, Australia \\ B Centre for Astronomy, Solar Radiation and Climate, University of Southern Queensland, \\ Toowoomba, QLD 4350, Australia \\ ${ }^{\mathrm{C}}$ Corresponding author. Email: irgeo@ozemail.com.au
}

Received 2007 June 13, accepted 2008 April 28

\begin{abstract}
We present evidence to show that changes in the Sun's equatorial rotation rate are synchronized with changes in its orbital motion about the barycentre of the Solar System. We propose that this synchronization is indicative of a spin-orbit coupling mechanism operating between the Jovian planets and the Sun. However, we are unable to suggest a plausible underlying physical cause for the coupling. Some researchers have proposed that it is the period of the meridional flow in the convective zone of the Sun that controls both the duration and strength of the Solar cycle. We postulate that the overall period of the meridional flow is set by the level of disruption to the flow that is caused by changes in Sun's equatorial rotation speed. Based on our claim that changes in the Sun's equatorial rotation rate are synchronized with changes in the Sun's orbital motion about the barycentre, we propose that the mean period for the Sun's meridional flow is set by a Synodic resonance between the flow period $(\sim 22.3 \mathrm{yr})$, the overall 178.7-yr repetition period for the solar orbital motion, and the 19.86-yr synodic period of Jupiter and Saturn.
\end{abstract}

Keywords: sun: activity — sun: sunspots — sun: rotation — stars: planetary systems

\section{Introduction}

Attempts to link solar activity on the surface of the Sun to planetary influences have had a long and checkered history. The published research has shown that there is considerable circumstantial evidence suggesting a link between:

a) Sunspot activity and the movement of the Sun about the centre-of-mass (CM) of the Solar System (Jose 1965; Landscheidt 1981, 1999; Fairbridge \& Shirley 1987; Chárvátova 1990, 2000; Zaqarashvili 1997). Specifically, between the strength of the sunspot cycles and the rate of change of the orbital angular momentum of the Sun about the CM of the Solar System (Jose 1965).

b) Deviations in the rotation rate of sunspots and the synchronicity of the orbital periods of the Jovian planets (Javaraiah \& Gokhale 1995; Javaraiah 2003).

c) North-South asymmetries between solar sunspot activity and differential rotation produced by a spinorbit coupling between the Sun and the Jovian planets (Juckett 2000).

However, these efforts to link solar activity to planetary influences have suffered from three major problems:

a) The tides induced on the surface of the Sun by the planets are so small that it is difficult to image that they could have any significant influence on solar surface activity. This means that if there is any connection, it must be via a transfer of orbital angular momentum from the Jovian planets into spin angular momentum of the Sun. As yet, no one has come up with a plausible physical mechanism that would produce a spin-orbit coupling between the Sun and the Jovian planets.

b) While Jose (1965) has shown that there is a good temporal match between the variations seen in the orbital angular momentum of the Sun about the Solar System's Barycentre and the variations seen in the sunspot cycle, the match is not always exact, e.g. Jose (1965) had to introduce arbitrary changes to the phase of the solar cycles in order to force a match between the planetary driving mechanism and variation in the level of solar activity.

c) The instantaneous rate of change in the Sun's angular momentum about the barycentre of the Solar System repeats itself with a periodicity of $178.8 \mathrm{yr}$ (Jose 1965), so that there should be no pause in the planetary driving mechanism. How then do we explain the lull in solar activity that occurred during the Maunder minimum?

The purpose of this paper is to address the first two problems. The third problem concerning the Maunder minimum is addressed by Fairbridge \& Shirley (1987) and Chárvátova (1990, 2000) and so it will not be discussed in this paper.

In Section 2 we show how the Sun's orbital angular momentum about the CM of the Solar System periodically 
decreases and then increases at successive quadratures of Jupiter and Saturn.

In Section 3 we present evidence to show that there is a direct link between the decreases and increases in the Sun's orbital angular momentum about the CM of the Solar System, and the observed decreases and increases in the Sun's equatorial rotation speed. We believe that this link provides strong circumstantial evidence that there is a spin-orbit coupling mechanism operating between the Jovian planets and the Sun. We propose that it is these changes in the Sun's rotation speed that are responsible for variations in the speed of the meridional flow. We postulate that it is the planetary induced changes in speed of the meridional flow that control both the duration and strength of sunspot activity on the Sun's surface. Finally, in Section 4 we present our conclusions.

\section{The Sun's Orbital Angular Momentum}

Given the fact that the Sun is over 1000 times the mass of Jupiter, it is often assumed that the CM of the Solar System is located at the centre of the Sun. In fact, the centre of the Sun moves about the CM of the Solar System in a series of complex spirals with the distance between the two varying from 0.01 to 2.19 solar radii (Jose 1965). This motion is the result of the gravitational forces of the Jovian planets tugging on the Sun.

Jose (1965) quantified the motion of the Sun about the $\mathrm{CM}$ of the Solar System and showed that the time rate of change of the Sun's angular momentum about the instantaneous centre of curvature $\mathrm{d} P / \mathrm{d} T$, or torque, varies in a quasi-sinusoidal manner similar to the variation seen in the solar sunspot number. In fact, Jose (1965) found that the temporal agreement between variations in $\mathrm{d} P / \mathrm{d} T$ and the solar sunspot number were so good that it strongly hinted that there was a connection between the planetary induced torques acting on the Sun and sunspot activity. However, he did not fully explain how this connection worked.

Zaqarashvili (1997) proposed that the ellipticity of the orbit of the Sun about the CM of the Solar System was responsible for periodic differential rotations within the solar interior and that these internal motions governed the properties of the solar sunspot cycle. He made the simplifying assumption that Sun's path about the CM of the Solar System was solely determined by Jupiter. In this case, the Sun moves about the CM of the Solar System in a slightly elliptical orbit $(e=0.048)$ with a semi-major axis of 1.08 solar radii and a period of $11.86 \mathrm{yr}$, i.e. the Sun rotates about a point located just above the solar surface.

Zaqarashvili's model made the first tentative steps to identify a spin-orbit coupling mechanism that might explain how planetary induced torques could produce periodic differential rotation within the solar interior (Zaqarashvili 1997). Unfortunately, his assumption, that the Sun's motion about the CM of the Solar System is primarily determined by Jupiter, is far too simplistic.

Figure 1 shows a typical orbit of the Sun about the CM. It is not the simple ellipse you would expect if gravitational

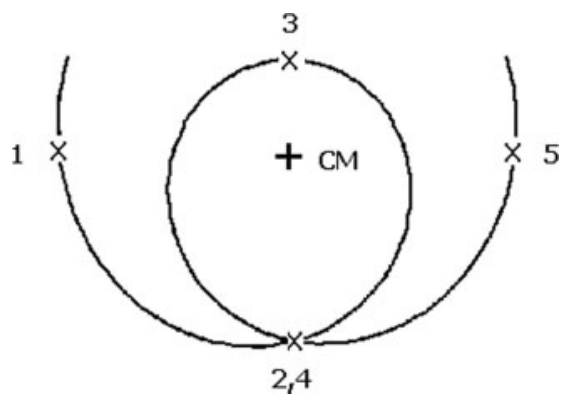

Figure 1 A diagram showing a typical orbit for the Sun about the CM of the Solar System, with the position of the Sun marked by an ' $\mathrm{X}$ ' at the times when Jupiter and Saturn are in opposition (1), first quadrature (2), conjunction (3), second quadrature (4), and opposition (5).

effects of Jupiter dominated the Sun's motion. The Sun's orbit about the CM deviates from an ellipse primarily because of the added influence of Saturn.

Obviously, when Jupiter is at inferior conjunction as seen from Saturn, i.e. the planets are on the same side of the Sun $^{1}$, the Sun will be at its greatest distance from the $\mathrm{CM}$; when Jupiter is at superior conjunction, as seen from Saturn, i.e. the planets are on opposite sides of the $\mathrm{Sun}^{2}$, the Sun will be closest to the CM. Similarly, when the planets are in quadrature, the Sun's distance from the CM will be roughly the same and somewhere in between these two extremes.

This point is highlighted in Figure 1 where we have marked a set of sequential events concerning the orbits of Jupiter and Saturn along the Sun's orbit about the CM. Jupiter and Saturn start in opposition at (1), first quadrature at (2), conjunction at (3), second quadrature at (4) and finally back to opposition at (5).

The net effect of adding the gravitational influence of Saturn to that of Jupiter upon the Sun's orbit about the CM is as follows:

a) The times at which the Sun experiences maximum torque $(\mathrm{d} P / \mathrm{d} T)$ as it moves around the $\mathrm{CM}$ of the Solar System, corresponds very closely with the times of quadrature for Jupiter and Saturn (Jose 1965) i.e. points (2) and (4) in Figure 1.

b) Similarly, the times at which the torque acting on the Sun is zero (this also the time at which the torque acting on the Sun is most rapidly changing) correspond very closely with the times of opposition and conjunction of Jupiter and Saturn, i.e. points (1), (3), and (5) in Figure 1.

Every $9.9 \pm 1.0 \mathrm{yr}$, the planet Saturn is in quadrature with the planet Jupiter (i.e. the angle between Saturn and Jupiter, as seen from the Sun, is $90 \mathrm{deg}$ ). Figure 2 shows the orbital configuration of a quadrature of Jupiter and Saturn

\footnotetext{
${ }^{1}$ In an inferior conjunction, the superior planet (Saturn) is 'in opposition' to the Sun, as seen from the inferior planet (Jupiter), and so we will refer to this as Jupiter and Saturn being in opposition.

${ }^{2}$ When Jupiter is at superior conjunction as seen from Saturn, we will refer to this as Jupiter and Saturn being in conjunction.
} 


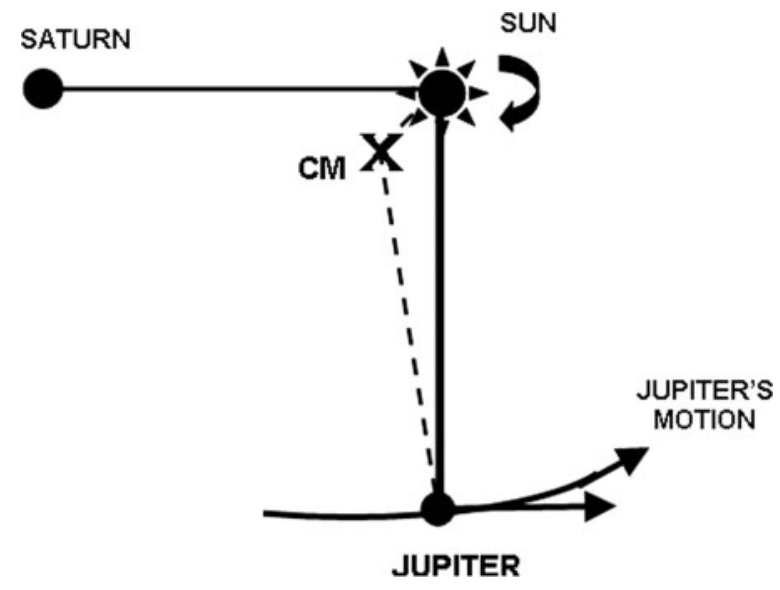

Figure 2 The Quadrature Effect when Saturn follows Jupiter: The gravitational pull of the Sun on Jupiter increases Jupiter's orbital speed. Conversely, the gravitational pull of Jupiter on the Sun decreases the Sun's orbital speed about the CM of the Solar System.

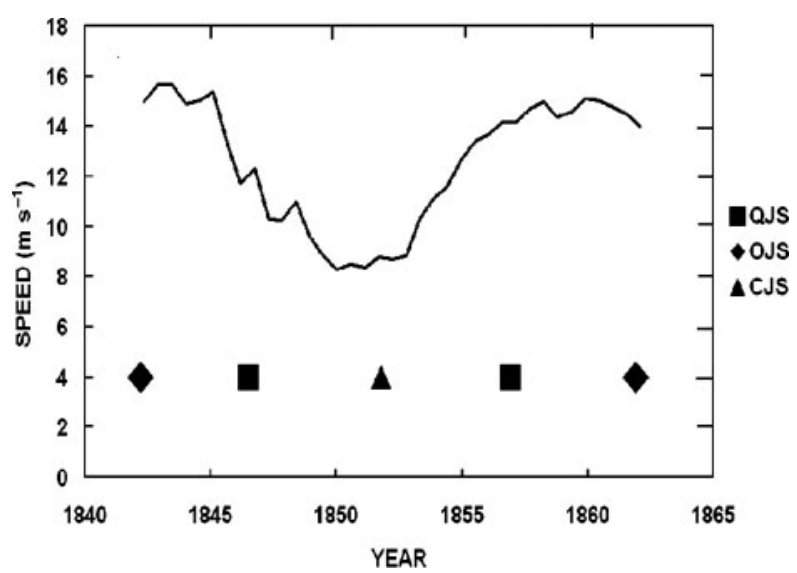

Figure 3 A plot of the speed of the Sun along its orbit about the $\mathrm{CM}$ of the Solar System, between the oppositions of Jupiter and Saturn in 1842.2 and 1861.9. OJS: Opposition of Jupiter and Saturn, QJS: Quadrature, CJS: Conjunction. N.B. the solid curve in figure 3 should be smooth; its jagged nature is simply an artifact that is produced by the digitization of Jose's original data by the author.

when Saturn follows Jupiter in its orbit. Referring to this diagram, we see that Saturn drags the CM of the Solar System off the line joining the planet Jupiter to the Sun. As a result, the gravitational force of the Sun acting upon Jupiter speeds up its orbital motion about the CM. At the same time, the gravitational force of Jupiter acting on the Sun slows down the orbital speed of the Sun about the CM.

However, the reverse is true at the next quadrature, when Saturn precedes Jupiter in its orbit. In this planetary configuration, the mutual force of gravitation between the Sun and Jupiter slows down Jupiter's orbital motion about the CM and speeds up the Sun's orbital motion about the CM. Hence, the Sun's orbital speed about the CM should periodically decrease and then increase as you move from one quadrature to next (Jose 1965).

The curves published by Jose (1965) showing the motion of the Sun about the CM can be used to directly measure the speed of the Sun along its orbital path. Figure
3 shows the speed of the Sun along its orbit, between the oppositions of Jupiter and Saturn in 1842.2 and 1861.9.

Superimposed on Figure 3 are symbols showing the syzygies and quadratures of Jupiter and Saturn. This figure clearly shows that our prediction about the Sun's orbital speed is indeed correct. In this plot, we see that the speed of the Sun almost halves (from $\sim 16$ to $8 \mathrm{~m} \mathrm{~s}^{-1}$ ) over the period from 1842 to 1850 , roughly centred on the time of the first quadrature in 1846.5. And then after reaching a minimum near conjunction in 1851.8 , the speed almost doubles (from $\sim 8$ to $15 \mathrm{~m} \mathrm{~s}^{-1}$ ) over the period from 1850 to 1860 , again roughly centred on the time of quadrature in 1856.9 .

It is important to note that it is not just the speed of the Sun about the CM that changes between oppositions but also the Sun's orbital radius about the CM as well. During the eight-year time period between 1842 and 1850, for example, the Sun's orbital radius about the CM changed from $\sim 2 R_{\mathrm{O}}$ to almost zero. This means that there was an overall decrease in the Sun's angular momentum about the $\mathrm{CM}$ of $\sim 4.5 \times 10^{40} \mathrm{Nms}$. Similarly, between 1850 and 1860 , the Sun's orbital radius about the CM increased from zero to $1.5 R_{\mathrm{O}}$, resulting in an increase of the Sun's angular momentum about the $\mathrm{CM}$ of $\sim 3.2 \times 10^{40} \mathrm{Nms}$.

Thus, the torque acting on the Sun about the CM starts out at zero at opposition, reaches a minimum value at the first quadrature (when Saturn follows Jupiter), returns to zero at the following conjunction, reaches a maximum at the second quadrature (when Saturn precedes Jupiter), and the finally returns to zero when the planets return to opposition. Variations in the torque of this nature produces a strong breaking of the Sun's orbital motion about the CM near the time of first quadrature, accompanied by a significant decrease in the Sun's angular momentum. This is followed by a strong acceleration of the Sun's orbital motion about the CM near the time of the second quadrature, accompanied by a comparable increase in the Sun's angular momentum.

Published plots of the torque $(\mathrm{d} P / \mathrm{d} T)$ acting on the Sun, where the torque is measured about the instantaneous centre of curvature of the Sun's orbit about the CM (Jose 1965), show that this is in fact what happens to the Sun. Jose's (1965) plots show the torque varying in a quasi-sinusoidal manner, starting out at zero at opposition, reaching a minimum at the first quadrature, returning to zero at the following conjunction, reaching a maximum at the second quadrature and finally returning to zero at the next opposition. The average time taken for this cycle to repeat itself is simply set by the synodic period of Jupiter and Saturn, i.e. $19.86 \mathrm{yr}$.

Figure 4 shows a plot of $\mathrm{d} P / \mathrm{d} T$, derived from data in the paper by Jose (1965), for one cycle between 1901.6 and 1920.9 (solid line). Superimpose on this plot (dashed line) is a sinusoidal function with a period equal to the time between the consecutive oppositions at 1901.8 and 1921.8 (i.e. $20.0 \mathrm{yr}$ ) and an amplitude chosen to match of the first minimum. 


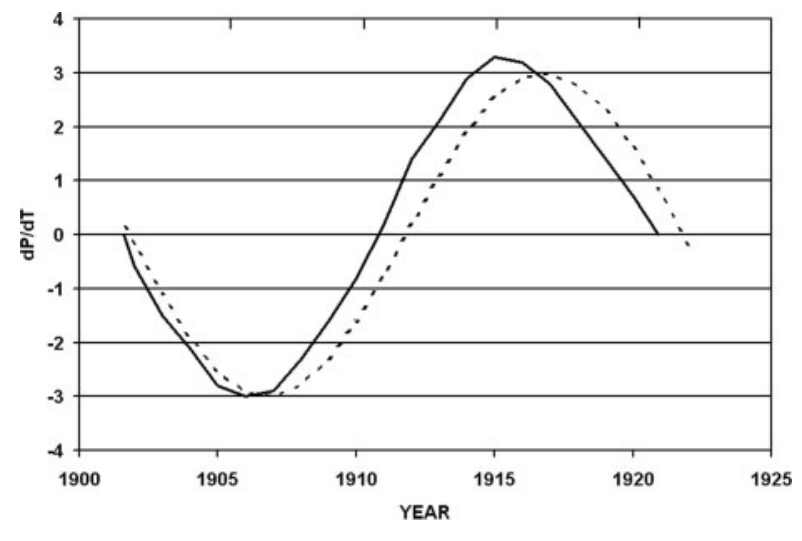

Figure 4 A plot of $\mathrm{d} P / \mathrm{d} T$ derived from data published in the paper by Jose (1965) for one cycle between 1901.6 and 1920.9 (solid line). Superimpose on this plot (dashed line) is a sinusoidal function with a period equal to the time between the consecutive oppositions at 1901.8 and 1921.8 (i.e. $20.0 \mathrm{yr}$ ) and an amplitude chosen to match of the first minimum.

We can see from Figure 4 that it is a reasonable first approximation to say that the Sun's orbital motion around the CM of Solar System is being driving by a torque $(\mathrm{d} P / \mathrm{d} T)$ that is varying sinusoidally with a period of $\sim 19.9$ yr. However, there are other weaker perturbing influences that are advancing or retarding the times for the maxima, minima and zero points of $\mathrm{d} P / \mathrm{d} T$, compared to the cardinal planetary configurations. These weaker perturbations are primarily caused by the combined gravitational influences of Neptune and Uranus (Fairbridge \& Shirley 1987). However, in this paper, we will ignore these effects upon the motion of the Sun, in order to simplify our arguments. Nevertheless, future papers on this topic will have to account for these important perturbing effects.

\section{Evidence for a Spin-Orbit Coupling}

\subsection{A Synodic Resonance?}

The Schwabe cycle was discovered by Samuel Heinrich Schwabe in 1843, when he noticed that the number of sunspots seen on the Sun's surface increased and then decreased over a period of about $10 \mathrm{yr}$ (Schwabe 1843). In reality, the actual cycle length of the Schwabe cycle can vary from about 9 to $14 \mathrm{yr}$ (Rogers et al. 2006), although it does oscillates about a long-term average value of about $11.1 \mathrm{yr}$.

Normally, the length of the sunspot cycle is measured from one solar minimum to the next. The simple reason for this is that it is easier to identify the time of solar minimum more precisely than the time of solar maximum. Indeed, there are some solar to that have two distinct peaks (e.g. cycle 23) making it difficult to identify the actual time of maximum solar activity.

By convention, the time of solar minimum is calculated using the smoothed sunspot number. The smoothed sunspot number is obtained by taking the 12-month moving average of the monthly sunspot number. It is generally agreed that solar minimum takes place when the smoothed sunspot number reaches a minimum. Strictly speaking, this means that solar cycle lengths are not well determined prior to January 1749 , since only yearly sunspot numbers were systematically reported prior this date. It is possible, however, to use either the annual Wolf (Zurich) sunspot number or the group sunspot number, to make reasonable estimates of the times of solar minimum back until the start of solar cycle -4 in 1698.0.

If we measure the average length of the Schwabe cycles, from one solar minimum to the next, for cycle numbers -4 to 23 , we get $11.1 \pm 1.2 \mathrm{yr}$ (Rogers et al. 2006), using Wolf sunspot numbers and $11.0 \pm 1.1 \mathrm{yr}$ (Usoskin \& Mursula 2003), using group sunspot numbers.

George Hale discovered that it took two Schwabe cycles (or one Hale cycle) for the magnetic polarity of sunspot pairs to reverse and then return to their original state (Hale 1908). This means that the sunspot activity cycle actually takes roughly $22 \mathrm{yr}$ to repeat itself.

Again, if we measure the average length of the Hale cycle, for solar cycle pairs, starting at cycle number -4 and finishing at cycle number 23 , we get $22.0 \pm 2.0 \mathrm{yr}$ (Rogers et al. 2006) using Wolf sunspot numbers and $22.1 \pm 1.9 \mathrm{yr}$ (Usoskin \& Mursula 2003), using group sunspot numbers.

Now, there are two dominant 'fixed' time scales associated with the torques applied to the Sun by the Jovian planets. One is the 19.858-yr synodic period of Jupiter and Saturn. The other is the repetition time scale for the planetary torques as a whole, which is $178.8 \mathrm{yr}$ (Jose 1965). This raises the question as to why there is a near resonances between the synodic period of Jupiter and Saturn and a time period $(\sim 22.3 \mathrm{yr})$ that closely matches the long-term average length of the Hale cycle $(22.1 \pm 1.9 \mathrm{yr})$, such that:

$$
\frac{22.34 \times 19.859}{22.34-19.859}=178.8 \mathrm{yr}
$$

It seems very unlikely that this just happened by chance. A much more plausible explanation is that there is an 8:9 resonance between the average length of the Hale sunspot cycle and synodic period of Jupiter and Saturn, with eight Hale cycles equal to nine Synodic periods for Jupiter and Saturn equal to 178.8 yr (one de Vries, or Suese, Cycle).

Hathaway et al. (2003) claimed that the average length of the Hale sunspot cycle is set by the period of the meridional flow in the Sun's convective layer. If they are correct, then it possible that the mean period of the meridional flow ( $\sim 22.3 \mathrm{yr}$ ) is locked into a synodic like resonance with the 19.86-yr synodic period of Jupiter and Saturn and the 178.7-yr period for the planetary induced torques that are acting on the Sun.

\subsection{Planetary Syzygies}

The time of oppositions and conjunction (i.e. syzygies) for Jupiter and Saturn closely corresponds to the time at which the Sun experiences its maximum change in torque about the CM of the Solar System. Thus, if there is any connection between the planetary induced torques and the sunspot cycle, you might expect to see the effects of these periodic peaks in the shear force in the sunspot number record. 

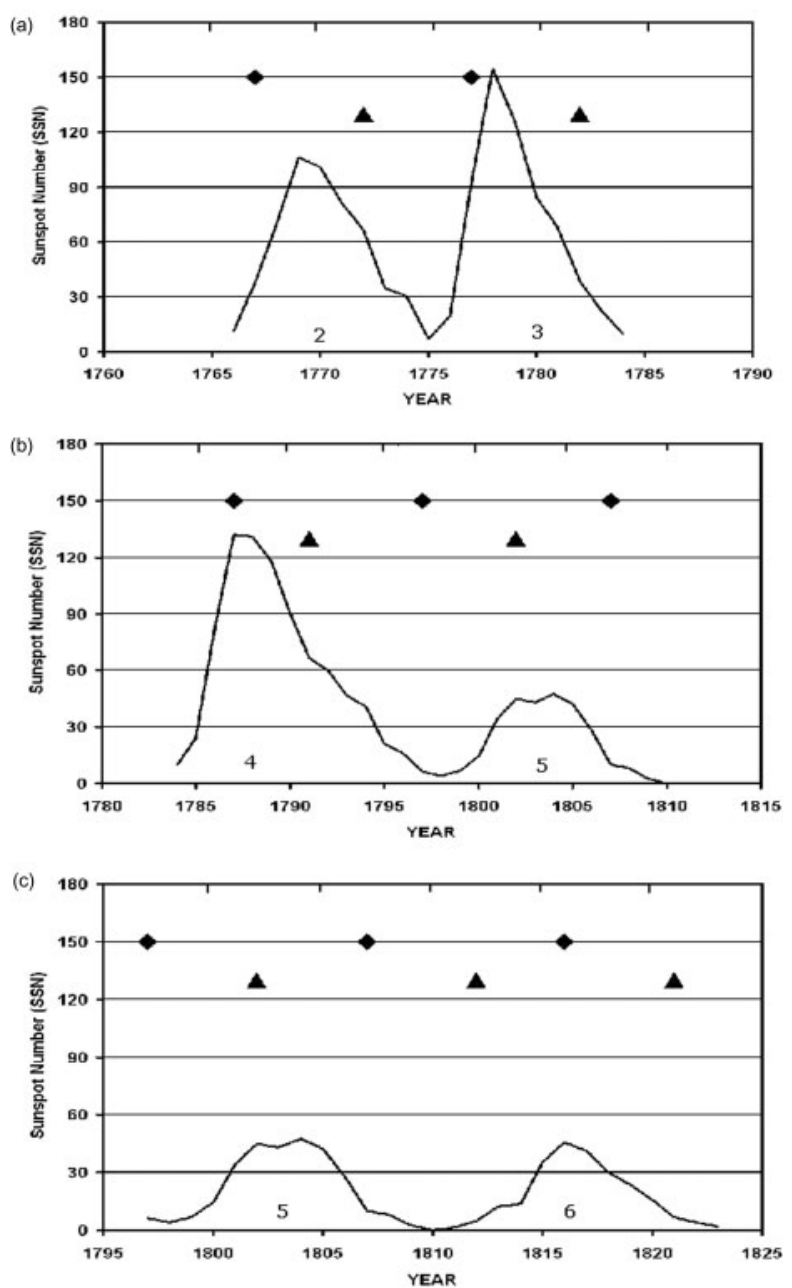

Figure 5 Figures 5a to 5c show the yearly International (Wolf) Sunspot Number from 1766 to 1823 . These graphs cover sunspot cycles 2 to 6 . Superimposed on these plots are filled triangles showing the times of the syzygies for Jupiter and Saturn and filled diamonds showing the quadratures.

Figures 5a to 5c show the yearly International (Wolf) Sunspot Number from 1766 to $1823^{3}$. These graphs cover sunspot cycles 2 to 6 . Superimposed on these plots are data points (filled triangles) showing the times of the syzygies for Jupiter and Saturn.

If you compare the annual solar sunspot number for this period with the times for the syzygies of Jupiter and Saturn, you find that the amplitude of a given solar cycle depends on whether the syzygy is before or after that solar maximum (Usoskin \& Mursula 2003). Note that for a given solar cycle, we are referring the syzygy that occurs between two consecutive minimums. In the rare case where there are two syzygies between consecutive solar minimums (i.e. cycles $-3,6$, and 14), we have chosen the one closest to the maximum.

If the syzygy occurs after the solar maximum then the strength of the solar maximum is normal. This is true for cycles 2, 3 and 4 which appear in Figures 5a and 5b. However, if the syzygy occurs before the solar maximum then

\footnotetext{
${ }^{3}$ World Data Centre for Sunspot Numbers, Belgium.
}

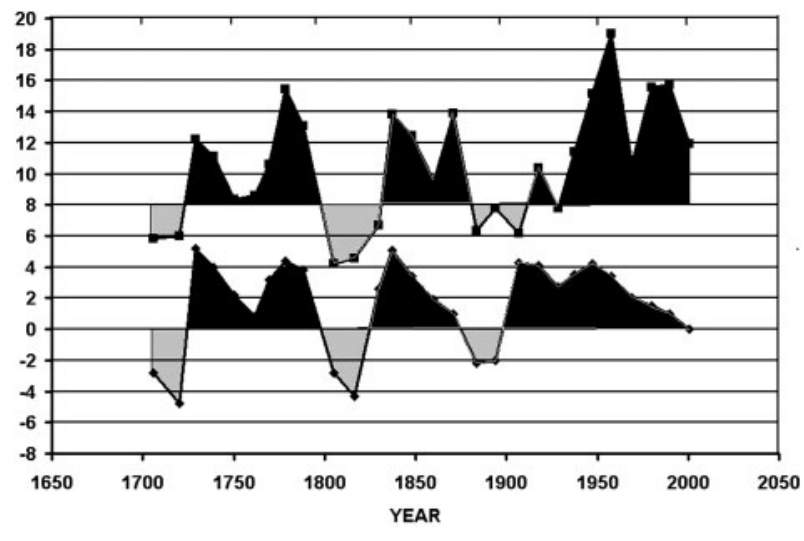

Figure 6 A comparison between the sunspot number at solar maximum (top curve) and the number of years that the syzygies of Jupiter and Saturn are ahead of the time of solar maximum (bottom curve). The sunspot numbers at solar maximum have been divided by 10 to produce a comparable scale.

the amplitude of the solar maximum collapses. This is true for cycles 5 and 6 which appear in Figures $5 \mathrm{~b}$ and $5 \mathrm{c}$.

Figure 6 shows that this phenomenon applies to all but a couple of the sunspot cycles. In Figure 6, we plot the Wolf Sunspot Number for the years of solar maximum between 1700 and 2004 (Usoskin \& Mursula 2003). We also plot the number of years that each syzygy of Jupiter and Saturn is ahead of the solar maximum ${ }^{4}$.

It is clear from a comparison of the two plots in Figure 6 that whenever the syzygies of Jupiter and Saturn occur after solar maximum, the Wolf Sunspot Number at solar maximum is above 80 . And whenever the syzygies occur before solar maximum, the Wolf Sunspot Number falls below 80 , i.e. when the driving force from the Jovian planets is in phase (or phase locked) with the solar cycle, then the amplitude of the cycles are normal. However, if there is a loss of phase locking (or phase drift), the amplitude of the cycles collapses.

The exception to this rule occurs for the first solar cycle following the reestablishment of phase lock. The peak sunspot number for these 'starter' cycles remains below 80 , despite the fact that phase lock has be reestablished.

This may simply mean that it takes at least one 11-yr (Schwabe) solar cycle to reestablish normal phase-locked solar activity. Note that Solar cycle -3 with a maximum at 1718.2 is probably the first cycle following the reestablishment of phase lock. There are two syzygies during this cycle, one at 1713.4 and the other at 1723.1. The difference in time between the times of syzygy and solar maximum is only $0.1 \mathrm{yr}$. This difference is well within the margin of error of the time measurements. Hence, it is possible that the time difference between syzygy and solar maximum might be $+4.9 \mathrm{yr}$ instead of $-4.8 \mathrm{yr}$, as shown in Figure 6 .

\footnotetext{
${ }^{4}$ Usoskin \& Mursula (2003) claim that there were solar maxima in the years 1720.2 and 1729.0. However, the Wolf Sunspot Numbers for these two cycles appear to peak at 1718.5 and 1727.0. Hence, for these cycles we have used the years for solar maximum quoted by Jose (1965) i.e. 1718.2 and 1727.5 .
} 


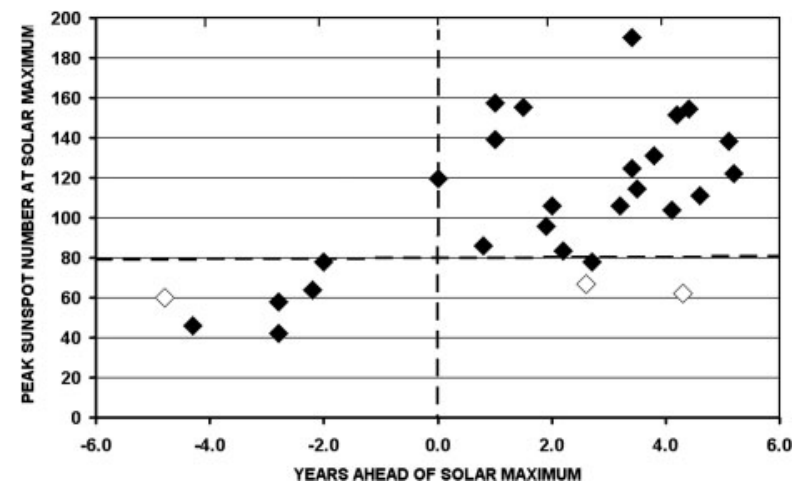

Figure 7 The peak sunspot number at solar maximum versus the number of years that syzygies of Jupiter and Saturn are ahead of or behind the times of solar maximum. The open diamond shaped points represent the first solar cycles following the reestablishment of phase lock.

A value of $+4.9 \mathrm{yr}$ would make this cycle fit the pattern seen during the other two phase collapses.

Between the late 17th century and 2004, the amplitude of the solar cycles has collapsed on three occasions because of a loss of phase lock. These losses of phase lock (or phase catastrophes) have occurred:

1. some time before cycle $-4(\sim 1680$ s?)

2. between cycles 4 and 5 in the early 1790 s

3 . between cycles 11 and 12 in the late $1870 \mathrm{~s}$

The amplitudes of the three cycles following each loss of phase lock are significantly reduced e.g. cycles 5, 6 and 7 following the loss of lock in the early 1790s and cycles 12,13 and 14 following the loss of lock in the late 1870s.

Another way to represent the data in Figure 6 is shown in Figure 7. Here we plot the peak sunspot number at solar maximum against the number of years that each syzygy of Jupiter and Saturn is ahead of the time of solar maximum. This plot clearly shows the two dominant modes of solar activity, i.e. the phase-locked and phase-drift modes.

\subsection{The Sun's Equatorial Rotation Rate}

While we have shown that the planetary induced torques acting on the Sun appear to influence the strength of the sunspot cycles, the evidence for this influence is indirect. It would be much better if we could show that there is a direct link between the regular decrease and then increase in the Sun's angular momentum about the CM of the Solar System and changes in the Sun's rotation rate. This would confirm that there is a coupling between the orbital angular momentum of the Solar System (primarily Jupiter's) and the spin angular momentum of the Sun.

In Section 2, we showed that the angular momentum of the Sun about the CM of the Solar System sequentially decreases, and then increases, between each opposition of Jupiter and Saturn. We also showed that these changes in angular momentum are in response to alternating toques that are applied to the Sun by the successive quadratures of Jupiter and Saturn (i.e. they produced by the quadrature effect).

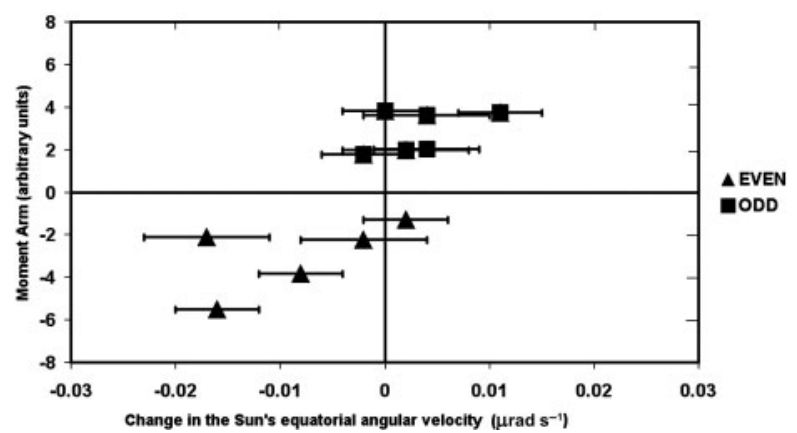

Figure 8 A plot of the moment arm of the torque for the quadrature nearest the maximum for a given cycle against the change in the average equatorial spin angular velocity of the Sun since the previous solar cycle (measured in $\mu \mathrm{rad} \mathrm{s}^{-1}$ ).

The question is, are there any systematic changes in the Sun's rotation rate in response to these changes in Sun's angular momentum about the CM?

One way to quantify the applied torques, is to use the moment arm (about the CM) of the gravitational force between the Sun and Jupiter, at the time of quadrature. Figure 8 shows the moment arm of the torque for the quadrature nearest the maximum for a given cycle, plotted against the change in the average equatorial (spin) angular velocity of the Sun since the previous solar cycle (measured in $\left.\mu \mathrm{rad} \mathrm{s}^{-1}\right)$. The equatorial $(\leq \pm 15 \mathrm{deg})$ angular velocities published by Javaraiah (2003) for cycles 12 to 23 have been used to determine the changes in the Sun's angular velocity (since the previous cycle) for cycles 13 to 23 .

Remarkably, Figure 8 shows that, whenever the Jovian induced torque acting on the Sun decreases the Sun's angular momentum about the CM, the Sun also experiences a decrease in its equatorial rotation rate. In a similar manner, whenever the Jovian induced torque increases the Sun's angular momentum about the CM, the Sun experiences an increase in its equatorial rotation rate. The only two exceptions to this rule are cycles 20 and 21 , although their deviations from the overall trend are within the uncertainties of the observation.

Interestingly, Javaraiah (2003) noted that since 1879 the equatorial rotation rate of the Sun has been significantly higher in the odd numbered solar cycles than the even numbered solar cycles, i.e. the equatorial solar rotation rate increases from even to odd solar cycles. This is highlighted in Figure 8, where we have used symbols to differentiate the five even numbered cycles from the six odd cycles. Interpreting this phenomenon in light of our quadrature model, we can see that it comes about simply because the quadratures of Jupiter and Saturn closest to the maxima of the odd number cycles have all been ones which increase the Sun's angular momentum about the $\mathrm{CM}$ of the Solar System. In contrast, the quadratures closest to the maxima of the even number cycles have all been ones which decrease the Sun's angular momentum about the CM.

Thus, the relationship shown in Figure 8 is the 'smoking gun' which provides us with a direct link between 
the planetary induced torques acting on the Sun and the observed changes in the Sun's equatorial rotation rate.

It is important to note, however, that while our data are consistent with the idea that there is a spin-orbit coupling between Jupiter and the Sun, it does not tell us anything about the true nature of the underlying mechanism that might be causing this coupling. However, we can use our data to place some useful constraints upon possible mechanisms.

A plausible spin-orbit coupling model must be able to explain the following facts:

1. At the time of the quadrature nearest sunspot maximum, if the Sun's orbital angular momentum (about the CM) decreases, then the Sun's equatorial rotation rate (averaged over the sunspot cycle) must also decrease when compared to its average rate for the previous cycle.

2. At the time of the quadrature nearest sunspot maximum, if the Sun's orbital angular momentum (about the CM) increases, then the Sun's equatorial rotation rate (averaged over the sunspot cycle) must also increase when compared to its average rate for the previous cycle.

3. The spin-orbit coupling mechanism is synchronized with the times of quadrature for Jupiter and Saturn. This does not imply that the mechanism is a direct result of the planetary quadratures, only that it must be intimately related to the configuration of the two largest Jovian planets.

4. Between successive oppositions of Jupiter and Saturn, $\sim 3$ to $5 \times 10^{40} \mathrm{Nms}$ of orbital angular momentum (about the CM) is transferred back and forth between Jupiter and the Sun. In addition, the data in Figure 8 indicates that the average change in the Sun's equatorial angular velocity, from one cycle to the next, is $0.0062 \mu \mathrm{rad} \mathrm{s}^{-1}$ (Javaraiah 2003). If the whole of the Sun were to change its angular velocity by this much, this would lead to a change in Sun's rotational angular momentum of $3.5 \times 10^{38} \mathrm{Nms}$, when measured between successive sunspot cycles (Cox 2000). This would mean that, over one Jupiter-Saturn synodic period, $0.7-1.2 \%$ of the total orbital angular momentum transferred back and forth between the Sun and Jupiter would have to be subtracted from, and then added to, the rotational angular momentum of the Sun. Such an implausibly high percentage transfer would suggest that it's more likely that it is actually the outer layers of the Sun's convective zone, rather than the Sun as a whole, that is affected by periodic changes in rotational speed.

5. The solar convective zone (SCZ) contains roughly $2 \%$ of the Sun's total mass and it has an inner radius of $0.71 R_{\mathrm{O}}$. If we were to represent the $\mathrm{SCZ}$ as a torus with these mass and dimensions and we let it change its angular velocity by $0.0062 \mu \mathrm{rad} \mathrm{s}^{-1}$, then it would lead to a change in Sun's spin angular momentum of $4.5 \times 10^{37} \mathrm{Nms}$, when measured between successive sunspot cycles (Cox 2000). This would require that, over one Jupiter-Saturn synodic period, only
$0.09-0.15 \%$ of the total orbital angular momentum transferred back and forth between the Sun and Jupiter would have to be subtracted from, and then added to, the rotational angular momentum of the convective layers of the Sun.

It is interesting to note that the average change in the equatorial rotation rate between solar cycles of $0.0062 \mu \mathrm{rad} \mathrm{s}^{-1}$, corresponds to a change in speed at the Sun's equator of $4.3 \mathrm{~m} \mathrm{~s}^{-1}$. This is remarkable close to the amplitude of the torsional oscillations seen on the surface of the Sun of $\sim 6 \mathrm{~m} \mathrm{~s}^{-1}\left(0.0086 \mu \mathrm{rad} \mathrm{s}^{-1}\right)$ observed by Howe et al. (2000). This raises the intriguing possibility that the torsional oscillations in the outer layers of the Sun may be a product of the mechanism that is responsible for the spin-orbit coupling between Jupiter and the $\operatorname{Sun}^{5}$.

\section{Conclusions}

Jose (1965) quantified the motion of the Sun about the CM of the Solar System and showed that the time-rate of change of the Sun's angular momentum about the instantaneous centre of curvature $\mathrm{d} P / \mathrm{d} T$ varies in a quasisinusoidal manner similar to the variations seen in the solar sunspot number. In addition, he noted that the temporal agreement between variations in $\mathrm{d} P / \mathrm{d} T$ and the solar sunspot number was so good that it strongly suggested a connection between the planetary induced torques acting on the Sun and sunspot activity.

In this paper, we have followed Jose (1965) lead and looked for a possible links between the torques applied to the Sun by the Jovian planets and the level of sunspot activity. The evidence that we found to support such a link was both direct and indirect.

\subsection{Indirect Evidence for a Spin-Orbit Coupling}

We performed a detailed comparison between the timing of the syzygies of Jupiter and Saturn and the times of solar maximum for all solar cycles since 1700 . This comparison showed us that the peak amplitude of a given solar cycle depends on whether the syzygy that is nearest in time to solar maximum is before or after that maximum. If the syzygy of Jupiter and Saturn nearest solar maximum occurs after solar maximum, the peak wolf sunspot number at solar maximum is above 80 . We find that the Sun remains in this 'normal' state for extended periods ranging from 55 to $115 \mathrm{yr}$. At the end of this extended period of 'normal' activity, the solar cycle appears to suffer a phase catastrophe, with the syzygy nearest the next solar maximum occurring before that maximum. When this happens, the peak amplitude of the sunspot cycle collapses, falling below a peak wolf sunspot number of 80. The sunspot cycle remains in this collapsed state for approximately one Hale cycle before returning its 'normal'

\footnotetext{
${ }^{5}$ Another interesting point is that the typical change in the Sun's angular momentum about the $\mathrm{CM}$ from one syzygy to the next is $\sim 3$ to $5 \times 10^{40} \mathrm{Nms}$. This is virtually the same as the total rotational angular momentum of the Sun's convective zone which is $\sim 2$ to $3 \times 10^{40} \mathrm{Nms}$.
} 
state. We also note that when the 'normal' state is reestablished, the strength of the first Schwabe cycle remains subdued.

This is exactly the type of behaviour you would expect to see if there is a drifting phase locked resonance between the 19.86-yr period of the planetary induced torques and the 22.3-yr period for the level of sunspot activity.

In this model, when the syzygies of Jupiter and Saturn occur after solar maximum, the planetary torques acting on the Sun are in phase (or phase locked) with the solar cycle, producing a peak Wolf sunspot number at solar maximum that is 'normal'. However, the difference in the length of the synodic period of Jupiter and Saturn (19.86 yr) and the length of the Hale Cycle $(22.3 \mathrm{yr})$ ensures that the resonance lock is broken once the two cycles drifted out of phase with each other by half a synodic period ( $\sim 9.9 \mathrm{yr}$ ). This would take $\sim 90 \mathrm{yr}$ i.e. four Hale cycles $(4.0 \times 22.3=89.2 \mathrm{yr})$ or four and a half synodic periods $(4.5 \times 19.86=89.4 \mathrm{yr})$.

The breaking of the phase lock is marked by a phase catastrophe. After this event, the syzygy of Jupiter and Saturn nearest solar maximum occurs before the next solar maximum. In this case, the planetary torque acting on the Sun is out of phase with the solar cycle, resulting in a collapse of the peak wolf sunspot number. Lose of phase lock initiates a phase drift between the planetary driving mechanism and the sunspot cycle that continues until phase lock is reestablished. The rate of phase drift between the two cycles is enhanced by a significant increase in the length of the collapsed sunspot cycles ( $\sim 2 \mathrm{yr}$ ) compared to the normal cycles. This leads to the reestablishment of phase-lock after only one Hale Cycle.

Based on the premise that the period of the meridional flow in the convective zone of the Sun determines the length of the solar cycle, we propose that the mean period for this flow $(\sim 22.3 \mathrm{yr})$ is determined by a Synodic (phase locked) 'resonance' between the flow period, the 178.7-yr period of the planetary induced torques on the Sun and the $19.86-y r$ synodic period of Jupiter and Saturn. This is an 8:9 resonance, with eight Hale cycles equal to nine synodic periods of Jupiter and Saturn equal to $178.8 \mathrm{yr}(\sim$ one de Vries, or Suese, Cycle).

We propose that every 55 to $115 \mathrm{yr}$, the planetary driving mechanism for the solar cycle experiences a phase catastrophe that subdues the level of solar activity for roughly one Hale cycle. The timing of the phase catastrophe is a quasi-stochastic process that is loosely set by the temporal phase difference between the length of the Hale Cycle ( $\sim 22.3 \mathrm{yr})$ and the synodic period of Jupiter and Saturn (19.86 yr). The quasi-stochastic nature in the timing of the phase catastrophe comes about simply because neither of the two interacting cycles has a fixed length. The length of the solar sunspot cycle can vary from 8.7 to $12.7 \mathrm{yr}$ (Usoskin \& Marsula 2003), while the length of the synodic period of Jupiter and Saturn can vary between 18.9 and 20.6 yr. However, on average, we expect the phase catastrophes to reoccur roughly every $90 \mathrm{yr}$ ( $\sim$ one Gleisberg cycle).

\subsection{Direct Evidence for a Spin-Orbit Coupling}

From a study of the motion of the Sun about the CM of the Solar System, we find that the torque acting on the Sun about the CM starts out at zero at opposition, reaches a minimum value at the first quadrature (when Saturn follows Jupiter), returns to zero at the following conjunction, reaches a maximum at the second quadrature (when Saturn precedes Jupiter) and then finally returns to zero when the planets return to opposition. Variations in the torque of this nature produces a strong breaking of the Sun's orbital motion about the CM near the time of first quadrature, accompanied by a significant decrease in the Sun's angular momentum. This is followed by a strong acceleration of the Sun's orbital motion about the CM near the time of the second quadrature, accompanied by a comparable increase in the Sun's angular momentum.

Using data published by Javaraiah (2003) for the equatorial $(\leq \pm 15 \mathrm{deg})$ spin rate of the Sun for sunspot cycles 12 to 23 (i.e. data from 1879 to 2002), we show that whenever the Jovian induced torque acting on the Sun decreases the Sun's angular momentum about the CM, the Sun also experiences a decrease in its equatorial rotation rate. And, whenever the Jovian induced torque increases the Sun's angular momentum about the CM, the Sun experiences an increase in its equatorial rotation rate. This result provides us with direct observational evidence that there is a spinorbit coupling between the orbital angular momentum of Jupiter and the spin angular momentum of the Sun.

While our data is consistent with the idea that there is a spin-orbit coupling between the Jupiter and the Sun, it does not tell us the exact nature of the mechanism that causes this coupling. However, we have used our data to place some constraints on the possible spin-orbit coupling mechanisms.

A plausible spin-orbit coupling model must be able to explain the following:

1. At the time of the quadrature nearest sunspot maximum, if the Sun's orbital angular momentum (about the CM) decreases/increases, then the Sun's equatorial rotation rate (averaged over the sunspot cycle) decreases/increases, when compared to its average rate for the previous cycle.

2. The spin-orbit coupling mechanism is synchronized with the times of quadrature for Jupiter and Saturn.

3. If the whole of the Sun partakes in the observed periodic changes in the equatorial rotation rate, then over one Jupiter-Saturn synodic period, $0.7-1.2 \%$ of the total orbital angular momentum transferred back and forth between the Sun and Jupiter would have to be subtracted from, and then added to, the rotational angular momentum of the Sun.

4. If, as is more likely, it is actually the outer $2 \%$ of the Sun's mass, rather than the Sun as a whole, that are affected, then over one Jupiter-Saturn synodic period, approximately $0.09-0.15 \%$ of the total orbital angular momentum transferred back and forth between the Sun and Jupiter would have to be subtracted from, and 
then added to, the rotational angular momentum of the surface layers of the Sun.

We also found that the average change in the Sun's equatorial rotation speed is $4.3 \mathrm{~m} \mathrm{~s}^{-1}$. This is remarkable close to the velocity amplitude of the torsional oscillations seen on the surface layers of the Sun. Thus, it raises the possibility that the torsional oscillations seen in the outer layers of the Sun may be a product of the spin-orbit coupling between Jupiter and the Sun.

\subsection{Important Consequences of the Resonance Model}

Interestingly, the Sun's solar cycle has been in the phase locked mode for the last $105 \mathrm{yr}$ (1900-2005) and the indications are that it is about to suffer another phase catastrophe in the later part of cycle 24 (i.e. the solar cycle that will peak in $2011-2012$ ). If this is the case, then we should expect that in the two decades following the phase catastrophe, the world's mean temperature should be noticeably cooler i.e. the cooling should start in the late 2010s. This claim is based on the precedent that there were noticeable decreases in the world's mean temperature following the last two phase catastrophes. The cool period know as the Dalton Minimum (1800-1820) that followed the phase catastrophe in the early 1790s and a similar cool period called the Victorian Minimum (1880-1900) that followed the phase catastrophe in the late 1870s.

Finally, another important consequence the synodic (phase locked) resonance model is that that any solar type main sequence stars that exhibits solar cycles similar to the Sun must have at least two Jovian planets orbiting the star, such that their synodic period is comparable to the star's solar cycle length. This opens up the possibility that long term HK observations of magnetic activity in solar type stars could be used as an effective method for detecting Jovian planets orbiting these stars.

\section{Acknowledgments}

The lead author would like to thank Ian A. Wilson, Marion N. Wilson and the Wilson family for making the preparation of this paper possible.

\section{References}

Chárvátova, I., 1990, BAICz, 41, 56

Chárvátova, I., 2000, AnG, 18, 399

Cox, A. N., 2000, Allen's Astrophysical Quantities (4th Edition; New York: AIP Press; Springer)

Fairbridge, R. W. \& Shirley, J. H., 1987, SoPh, 110, 191

Hale, G. E., 1908, ApJ, 28, 315

Hathaway, D. H., Nandy, D., Wilson, R. M. \& Reichmann, E. J., 2003, ApJ, 589, 665

Howe, R., Christensen-Dalsgaard, J., Hill, F., Komm, R. W., Larsen, R. M., Schou, J., Thompson, M. J. \& Toomre J., 2000, ApJ, 533, L163

Javaraiah, J., 2003, SoPh, 212, 23

Javaraiah, J. \& Gokhale, M. H., 1995, SoPh, 158, 173

Jose, P. D., 1965, AJ, 70, 193

Juckett, D., 2000, SoPh, 191, 201

Landscheidt, T., 1981, J. Interdiscipl. Cycl. Res., 12, 3

Landscheidt, T., 1999, SoPh, 189, 413

Rogers, M. L., Richards, M. T. \& Richards, D., 2006 (astro-ph/ 0606426)

Schwabe, H., 1843, AN, 20, 495

Usoskin, I. G. \& Mursula, K., 2003, SoPh, 218, 319

Zaqarashvili, T., 1997, ApJ, 487, 930 